\title{
Electric current diagnostics in the magnetosphere of neutron stars
}

\author{
Alexander V. Stepanov ${ }^{1}$ and Valery V. Zaitsev ${ }^{2}$ \\ ${ }^{1}$ Pulkovo Observatory, Pulkovo chaussee 65, Saint Petersburg 196140, Russia \\ email: stepanov@gao.spb.ru \\ ${ }^{2}$ Institute of Applied Physics, Ulianova str. 46, Nizhny Novgorod 603950, Russia \\ email: za130@appl.sci-nnov.ru
}

\begin{abstract}
Active neutron stars - SGRs, reveal the high-quality QPOs at the 'pulsating tail' phase. We suggest diagnostics of the trapped fireball plasma, the source of high-frequency pulsations, using coronal seismology. The trapped fireball is represented as a set of current-carrying loops - equivalent of electric circuits. Our approach gives the following magnetosphere parameters in SGRs: an electric current of $(2-8) \times 10^{19} \mathrm{~A}$, magnetic field of $(0.6-2.7) \times 10^{13} \mathrm{G}$, and electrons density of $(1.3-6.0) \times 10^{16} \mathrm{~cm}^{-3}$. We show high-frequency QPOs can be self-excited for a smaller electric current than the maximum current and/or due to the parametric resonance.
\end{abstract}

Keywords. Stars: neutron, SGRs, flares, oscillations, current-carrying loops, diagnostics.

\section{Introduction}

Three giant flares of neutron stars (Table 1) with the energy release of $10^{44}-5 \times 10^{46}$ ergs, were accompanied by high-frequency (from tens to thousands $\mathrm{Hz}$ ) quasi-periodic X-ray pulsations (Barat et al. 1983; Strohmayer \& Watts 2006). A model of the highfrequency pulsations should explain not only their periods and excitation mechanism, but also their very high quality factor $Q \geqslant 10^{4}-10^{5}$. Currently, the most widespread models for high-frequency QPOs are based on global seismic oscillations of the magnetar. Some models of neutron stars implied the excitation of torsion oscillations of the crust with a shear during starquakes. However, Levin (2007) pointed out that the torsion modes of the crust decay very rapidly. Beloborodov \& Thompson (2007) proposed that non-linear oscillations of the production of electron-positron pairs emerge during the formation of a magnetar corona consisting of a set of magnetic loops. Nevertheless, current models are unable to explain the total set of the observed properties of QPOs with frequencies of 20 to $2400 \mathrm{~Hz}$ and their very high Q-factor. We represent the source of the pulsations a trapped fireball - as a set of current-carrying loops and use the analogy with the loop as an equivalent RLC-circuit (Alfven et al. 1967; Stepanov et al. 2012). The efficiency of our model is illustrated by the diagnostics of the magnetospheres of SGRs.

\section{The suggested approach: an equivalent electric (RLC) circuits}

The 'trapped fireball' can be represented as a set of current-carrying magnetic loops with various sizes whose eigen-frequencies, quality factors, and inductance are given by

$$
\nu=(2 \pi \sqrt{L C})^{-1}, Q=\frac{1}{R} \sqrt{\frac{L}{C}}, L=2 l\left(\ln \frac{4 l}{\pi r}-\frac{7}{4}\right) .
$$

Here, $R$ and $C$ are the resistance and capacitance of the coronal loop, and the inductance $L$ specified by the length $l$ and radius $r$ of a loop. Given the energy $E=L I^{2} / 2$ that 
Table 1. Pulsating tail properties in giant flares of SGRs and magnetosphere parameters.

\begin{tabular}{rrrr}
\hline & $\begin{array}{r}\text { SGR 0526-66 } \\
\text { March 5, 1979 }\end{array}$ & $\begin{array}{r}\text { SGR 1900+14 } \\
\text { August 27, 1998 }\end{array}$ & $\begin{array}{r}\text { SGR 1806-20 } \\
\text { December 27, 2004 }\end{array}$ \\
\hline Duration, s & $\sim 200$ & $\sim 400$ & $\sim 380$ \\
Energy, ergs & $3.6 \times 10^{44}$ & $1.2 \times 10^{44}$ & $1.3 \times 10^{44}$ \\
Main pulse period, s & 8.1 & 5.15 & 7.56 \\
QPO frequencies, Hz & 43 & $28,54,84,155$ & $18,26,30,93,150,625$, \\
& & & $720,976,1840,2384$ \\
Q-factor & $\sim 10^{4}$ & $\sim 10^{4}-10^{5}$ & $\sim 10^{4}-5 \times 10^{5}$ \\
& & Calculated parameters & $2 \times 10^{19}$ \\
Electric current, A & $8 \times 10^{19}$ & $3 \times 10^{19}$ & $6 \times 10^{12}$ \\
Magnetic field, G & $2.7 \times 10^{13}$ & $10^{13}$ & $1.3 \times 10^{16}$ \\
\hline Electron density, cm cm $^{-3}$ & $6 \times 10^{16}$ & $2 \times 10^{16}$ &
\end{tabular}

has been released in the flare tail, one may determine the current $I$ in the coronal loop and hence the coronal plasma density and the $\varphi$-component of the magnetic field. From the observed energy release power $W=R I^{2}$ one can find the resistance $R$ of a loop, while from the oscillations frequency the loop's capacity $C$ may be estimated, that is, the quality factor $Q$ of the oscillations. Let us illustrate the efficiency of the proposed model.

Pulsating tail of SGR 1806-20 on 27 December, 2004. The energy released in this flare was of the order of $5 \times 10^{46} \mathrm{erg}$, and the energy of the "ringing tail" was of the order of $10^{44}$ ergs. Taking into account the great variety of QPO frequencies (Table 1) we will suggest that the energy stored in an "average" loop in the course of "ringing tail" is roughly $10^{43} \mathrm{erg}$. Supposing $l=3 \times 10^{6} \mathrm{~cm}$ and $r=3 \times 10^{5} \mathrm{~cm}$, we can use Eqs. (1) to find its inductance $L \approx 5 \times 10^{-3}$ Henry. Assuming that the stored energy of an "average" loop, $E \approx 10^{43}$ ergs $=10^{36} \mathrm{~J}$, has been released we obtain the current $I=(2 E / L)^{1 / 2} \approx$ $2 \times 10^{19} \mathrm{~A}$, from which we estimate the $\varphi$-component of the magnetic field in the loop $B_{\varphi} \approx I / c r \approx 6 \times 10^{12} \mathrm{G}$. The electron-positron pairs density $n$ in the source can be obtained from the electric current $I=e n c S$ and the cross section of the coronal loop $S$ with the radius $r=3 \times 10^{5} \mathrm{~cm}$. For $I=1.8 \times 10^{19} \mathrm{~A}, n=1.3 \times 10^{16} \mathrm{~cm}^{-3}$, i.e., the Langmuir frequency $\nu_{p} \approx 1 \mathrm{THz}$ corresponds to the sub-mm wavelengths. The power of the energy release in the tail is of the order of $10^{41} \mathrm{erg} / \mathrm{s}$, i.e., for an "average" loop $W=R I^{2} \approx 10^{40} \mathrm{erg} / \mathrm{s}=10^{33} \mathrm{~W}$. The resistance of a loop is $R=W / I^{2} \approx 3 \times 10^{-6} \Omega$. One of the possible reasons for resistance may be the plasma wave instability driven by beams of high-energy electrons, accelerated in electric fields of the magnetar magnetosphere. The minimum $\left(\nu_{1}=18 \mathrm{~Hz}\right)$ and maximum $\left(\nu_{2}=2384 \mathrm{~Hz}\right)$ frequencies in QPOs allow the capacity of loops to be estimated from Eqs. (1): $C_{1} \approx 1.3 \times 10^{-2} \mathrm{~F}, C_{2} \approx 7 \times 10^{-7} \mathrm{~F}$. On the other hand, the capacity of a coronal loop may be presented as $C \approx \varepsilon_{A} S / l$, where $\varepsilon_{A}=c^{2} / V_{A}^{2}$ is the dielectric permeability of the medium for Alfven waves (Stepanov et al. 2012). It is known than in the magnetar corona $V_{A} \approx c$. Therefore, $\varepsilon_{A} \approx 1$ and we obtain $C \approx 10^{5} \mathrm{~cm}=10^{-7} \mathrm{~F}$, which is several times lower than that $C_{2}$ calculated from the Eq. (1). It is easy to see that with increasing $S$ as $l$ decreases (thick loop), the coincidence of the capacitance with $C_{2}$ and $C_{1}$ can be achieved. Applying the second relation from Eqs.(1), we find corresponding $Q$-factors $Q_{1} \approx 2 \times 10^{5}$ and $Q_{2} \approx 10^{7}$, which exceed the observed quality factors of QPOs. Note, that the coronal loop in SGRs is a system with compact parameters and Eqs.(1) can be applied. Indeed, oscillations of electric current should be in-phase in all points of a loop. On the other hands, variations of the current propagate along the loop with the Alfven velocity ( $\approx$ c for SGR). Therefore, the condition of phase coincidence $\nu \approx 20-2500 \mathrm{~Hz}<c / l \approx 10^{4} \mathrm{~Hz}$ is satisfied. 


\section{Excitation of high-frequency QPOs of the current in coronal loops}

For minor deviations of the electric current $|\tilde{I}| \ll I$, the equation for current oscillations in a loop can be presented as (Zaitsev et al. 2001):

$$
L \frac{d^{2} \tilde{I}}{d t^{2}}+\alpha\left(I^{2}-I_{\max }^{2}\right) \frac{d \tilde{I}}{d t}+\frac{\tilde{I}}{C}=0
$$

Eq. (2) indicates that oscillations will be excited for a smaller current than the maximum current in the giant pulse of the flare, $I<I_{\max }$. Parametric resonance can be another way for the excitation of magnetic loop oscillations. The electric current oscillations due to perturbations in the crust with the pumping frequency $\nu$ trough a parametric interaction with a coronal loop can trigger oscillations in the loop at the frequency $\nu$, at the subharmonics $\nu / 2$, and at the first upper frequency of the parametric resonance $3 \nu / 2$. The variations in coronal loop parameters can be described by the equation

$$
\frac{d^{2} y}{d t^{2}}+\nu_{0}^{2}(1+q \cos \nu t) y=0
$$

where $\nu_{0}$ is the frequency of the coronal loop eigen-mode. The parameter q defines the width of the zone near the parametric resonance frequency $\nu_{n}=n \nu / 2, n=1,2,3, \ldots$, namely $-q \nu_{0} / 2<\nu / 2-\nu_{0}<q \nu_{0} / 2$. The excitation occurs when the frequency of eigenoscillations of the loop $\nu_{0}$ falls on the first instability zone, i.e., it is close to $\nu / 2$. Thus for a coronal loop to be excited parametrically, it must have suitable size, density, and magnetic field. In the flare in SGR 1901+14 27, the QPO were excited due to parametric resonance: $\nu=54 \mathrm{~Hz}, \nu / 2=27 \mathrm{~Hz}$ (at the observed frequency $28 \mathrm{~Hz}$ ), $3 \nu / 2=81 \mathrm{~Hz}($ at the observed frequency $84 \mathrm{~Hz}$ ). Therewith the frequency width of the QPO peaks is about 1-5 Hz. Note that we obtain the observed frequencies $(\nu / 2=28 \mathrm{~Hz}$ and $3 \nu / 2=84 \mathrm{~Hz})$ with a high accuracy for $\nu$ equal to $56 \mathrm{~Hz}$ rather than $54 \mathrm{~Hz}$.

We present the source of QPOs, a trapped fireball, as a set of current-carrying loops - an equivalent electric (RLC) circuits. Nevertheless this phenomenological approach is quite effective diagnostic tool for magnetospheres very active neutron stars - Soft Gamma Repeaters. With this approach we determined the electric current in magnetospheres of SGR 0526-66, SGR 1806-20, and SGR 1900+14, electron density, and magnetic field $B<B_{q}=m^{2} c^{3} / \hbar e=4.4 \times 10^{13} \mathrm{G}$. It means that the physical processes in magnetar magnetospheres at the 'ringing tail' phase can be studied within non-quantum electrodynamics approach.

This work was partially supported by the RFBR grants No 12-02-00616a and 1102-00103a, the Program of Leading Scientific Schools (1625.2012.2 and 4185.2012.2), the RAS Program 'Non-steady-state Universe', and the Federal Goal-Oriented Program (code 2012-1.2.1-12-000-1012-010).

\section{References}

Alfven, H. \& Carlqvist, P. 1967, Solar Phys, 1, 220

Barat, S., Hayles, R. I., \& Hurley, K. et al. 1983, A\&A, 126, 400

Beloborodov, A. M. \& Thompson, C. 2007, ApJ, 657, 967

Levin, Yu. 2007, MNRAS, 377, 159

Stepanov, A. V., Zaitsev, V. V., \& Nakariakov, V. M. 2012, Coronal Seismology. Waves and Oscillations in Stellar Coronae (Wiley-VCH Verlag \& Co), 221

Strohmayer, T. E., Watts A. L. 2006, ApJ, 653, 593

Zaitsev, V. V., Kislyakov, A. G., \& Shkelev, E. I. 2001, Radiophys. Quantum Electron., 44, 756 